\title{
Cortical visual evoked potentials in very low birthweight premature infants
}

\author{
J Atkinson, S Anker, S Rae, F Weeks, O Braddick, J Rennie
}

Arch Dis Child Fetal Neonatal Ed 2002;86:F28-F31

See end of article for authors' affiliations

.....................

Correspondence to: Professor Atkinson, Visual Development Unit, Department of Psychology, University College London, Gower Street, London WCIE 6BT, UK; i.atkinson@ucl.ac.uk

Accepted

4 October 2001

\begin{abstract}
Objectives: To compare the age of onset of the pattern orientation reversal visual evoked potential (OR-VEP) in a group of very low birthweight (VLBW) premature infants with term infants matched for postconceptual age at testing. The OR-VEP measure is used as an indicator of visual cortical functioning because of the specificity of cortical neurones in showing sensitivity to changes of slant or orientation.

Design: Results are given for 24 VLBW infants, born at 24-32 weeks gestation weighing less than $1500 \mathrm{~g}$, and 31 infants born at term. The steady state evoked potential to a grating pattern reversing in orientation (between $45^{\circ}$ and $135^{\circ}$ ) at 4 reversals/second and 8 reversals/second was recorded. Results: The VLBW infants with normal neonatal ultrasound results (and normal neurological outcome at 3 years of age) showed a significant OR-VEP with a similar postnatal time course to the term infants. Four premature infants, showing appreciable abnormalities on ultrasound examination, did not show normal onset of the OR-VEP, and all had an abnormal neurological outcome.

Conclusions: This result can be taken to indicate that the onset of cortical function is similar in healthy preterm infants to term infants. The visual development of the premature infants was neither accelerated nor delayed as a result of their extra visual experience. The OR-VEP can be used as a prognostic indicator of early brain development alongside other neurological measures. It may also be a very early indicator of later neurological outcome.
\end{abstract}

in he development of a functioning cerebral cortex is one of the most important aspects of neurological development in the perinatal and postnatal period. Because the functions of visual cortex are relatively well understood, this functional development has been studied most fully in the visual domain. ${ }^{1-3}$ Over the past 20 years, we have developed a number of new tests (both behavioural and visual evoked potential (VEP) measures) to gauge the time course of normal visual development. ${ }^{4-6}$ Infants born before term, with very low birth weight, must enter the extrauterine environment with immature cortical function compared with term infants. On the one hand, this may deprive them of the optimal physiological environment in an important period of development. On the other hand, the development of the visual system may be accelerated by exposure to visual stimulation earlier than in term infants.

There have been many studies of visual function in infants born before term using behavioural measures and flash and simple pattern reversal VEP measures including the sweep method. ${ }^{7-17}$ Some have attempted to isolate particular components for analysis ${ }^{18}{ }^{19}$ and correlate them with abnormal brain development in premature infants. These measurements and behavioural measures have been used to predict neurological outcome. ${ }^{2021}$ In most of the studies cited above, estimates of development of visual acuity have been used. However, visual acuity reflects a number of optical, retinal, and central neural factors $^{22}$ and is not necessarily an indicator of cortical development; it is widely accepted that the acuity performance of the neonate is mediated by a subcortical route. ${ }^{2}$ VEPs, which have also been studied in infants before term, ${ }^{18} 202324$ generally originate in cortical structures. The presence of a VEP to a flash or pattern reversal stimulus implies that visual information is successfully activating the cortex. However, neural activity for these kinds of stimuli is elicited at earlier stages of the pathway from the eye to visual cortex. Such VEPs therefore do not reflect the maturity or otherwise of the processing that occurs within the visual cortex.
Orientation selective responses are known to originate in cortical cells of cats and monkeys but not in subcortical visual structures such as the lateral geniculate nucleus and the superior colliculus. Thus orientation selectivity can be used as a marker of cortical functioning. We have developed the method of orientation reversal VEP (OR-VEP) as a marker for this distinctive processing that occurs in visual cortex. The OR-VEP response emerges postnatally in normally developing term infants. ${ }^{25}{ }^{26}$ No significant VEP can be recorded to this change in orientation at relatively high reversal rates (4-8 reversals/second) in newborns, but by $2-3$ months nearly all normal infants show a significant OR-VEP. ${ }^{35}$ Using this onset time, we have already shown that the absence or abnormality of a significant OR-VEP correlates with abnormalities caused by perinatal events, observed on structural magnetic resonance imaging (MRI), in term infants with focal lesions and hypoxic-ischaemic encephalography. ${ }^{27-29}$

In this group of infants, MRI abnormalities seen in the basal ganglia correlated with delay or absence of the OR-VEP. ${ }^{30}$ Onset of the OR-VEP before five months after term also correlated with normal neurological outcome at 2 years of age in these term infants with focal lesions and hypoxicischaemic encephalography. ${ }^{31}$

Here we use this test to gauge the onset of cortical functioning in very premature infants. The purpose of the study was threefold: to find whether $(a)$ premature infants (corrected for gestational age) showed the same time course of visual development as term infants; $(b)$ the additional visual experience as the result of prematurity enhanced and accelerated visual development in premature infants (a matter addressed in previous studies $\left.{ }^{92}{ }^{33}\right)$; (c) these new tests could

Abbreviations: VEP, visual evoked potential; OR-VEP, orientation reversal VEP; MRI, magnetic resonance imaging; VLBW, very low birth weight. 
be used to predict later neurological problems in any of the premature group.

\section{METHODS}

\section{Study population}

Very low birthweight (VLBW) infants-that is, weighing less than $1500 \mathrm{~g}$ at birth-were recruited as volunteers from the special care baby unit of the Rosie Maternity Hospital, Cambridge. VLBW infants were born at 24-32 weeks gestation. In all, 32 VLBW infants were recruited, with data from 24 of them being reported here (data from the other eight were incomplete). Major abnormalities were found for four of these 24 infants on ultrasound examination: two showed periventricular leucomalacia and two had a massive intraventricular haemorrhage (which largely resolved in both cases). The two with periventricular leucomalacia had major neurological problems at follow up, one being diagnosed as "cortically blind". Three other VLBW infants showed minor germinal layer grade 1 haemorrhages, which resolved rapidly, and two showed transient flares. None of the latter five had appreciable neurological or visual problems during a three year follow up, although two were strabismic.

Thirty one control infants, volunteered by their parents, from the normal postnatal wards of the same hospital were tested. They were born without complications within two weeks of their due date. All control infants developed normal vision over the first three years of life.

The infants were entered into an extensive protocol receiving about seven visits between birth and 3 years of age for a series of behavioural and VEP tests. The OR-VEP results are the focus of this paper. All ages given are from the expected day of delivery (calculated from postmenstrual dates). Results are reported for 27 control infants and 20 VLBW infants who completed VEP testing at four days to six weeks after term (mean age 33.4 days for the control infants, 34.0 days for the VLBW infants) and 31 control and 24 VLBW infants who completed VEP tests at 12-17 weeks after term (mean age 100.4 days for control infants, 104.1 days for VLBW infants). There was no significant difference between the post-term ages at testing of the two groups for either test session. Some infants were tested a second time during a particular time period. Here we include the results from one testing session only, in which testing was completed with the infant in a good state throughout recording. For at least $80 \%$ of infants in each group at each age, this was the first time of testing with a particular stimulus condition. Any infant who did not complete testing within either the early time window (0-6 weeks after term) or the later time window (12-17 weeks after term) was not included in the analysis.

\section{Test procedure}

High contrast square wave gratings, fundamental spatial frequency $0.5 \mathrm{c} /$ degree, were presented on a video monitor at 40 $\mathrm{cm}$ viewing distance from the infant's eyes. A pattern sequence for OR-VEP was presented consisting of orientation reversals between obliquely oriented grating patterns at $45^{\circ}$ and $135^{\circ}$, at 4 reversals/second for the younger age group and 8 reversals/ second for the older. The orientation reversals were embedded in a sequence of random phase shifts at 25 per second. VEPs were bipolar recordings made from three scalp electrodes placed $1 \mathrm{~cm}$ above the inion and on the forehead (active electrodes) and at the vertex (ground). Steady state VEPs were analysed by signal averaging over 300 sweeps (2 reversals/sweep). The amplitude of the signal at the reversal rate $\left(\mathrm{F}_{2}\right.$, the second harmonic of the sweep frequency) was measured. The presence of a statistically significant VEP signal at this frequency was assessed by the "circular variance" test. ${ }^{34}$

\section{RESULTS}

Figure 1A shows the percentage of infants showing a significant VEP at 4 reversals/second at the younger age (0-6 weeks after

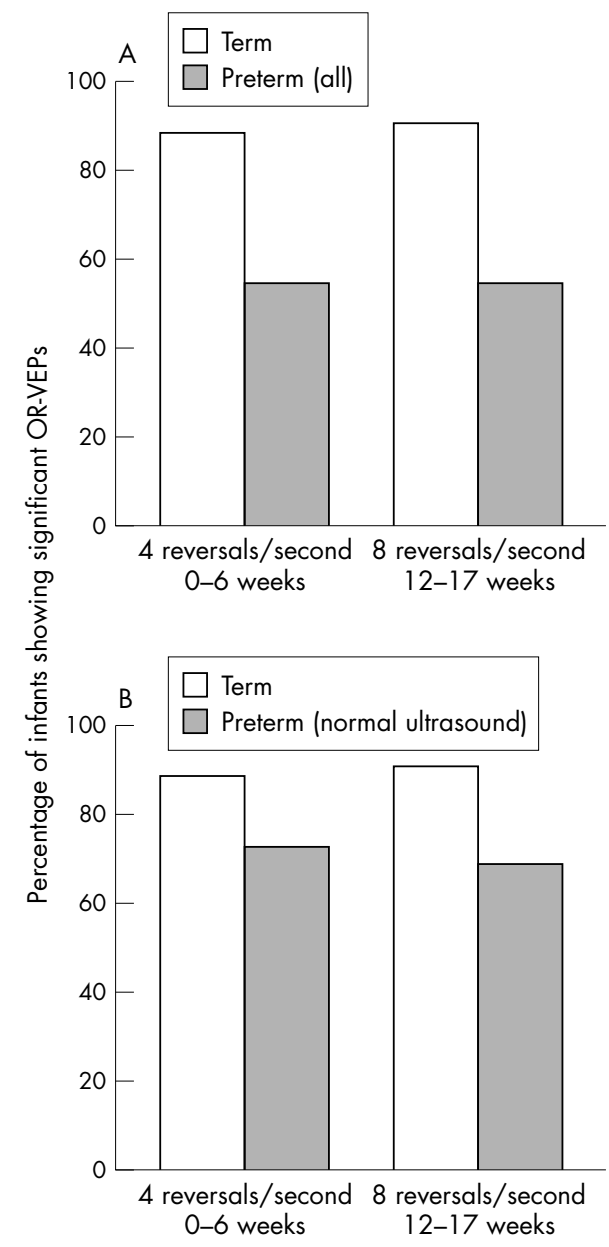

Figure 1 (A) Comparison of the entire group of very low birthweight premature infants (preterm), including those with abnormalities found by ultrasound, and the control group of infants born at term (term). The percentage of infants showing significant orientation reversal visual evoked potential (OR-VEP) at 4 reversals/second and 8 reversals/second is shown. The groups were matched for post-term age in two testing periods: $0-6$ weeks of age and 12-17 weeks of age. At both ages, there is a significant difference between the premature and term infants in the percentage showing a significant OR-VEP. (B) Comparison of the subset of very low birthweight premature infants (preterm) with normal ultrasound images, and the control group of infants born at term (term). At both ages, there is no significant difference between the premature and term infants in the percentage showing a significant OR-VEP, although slightly more term infants show the significant VEP response.

term) and 8 reversals/second at the older age (12-17 weeks after term). At each age, significantly more term infants in the control group than in the premature group showed the OR-VEP (younger groups: 24/27 control infants $v$ 11/20 VLBW infants, $\mathrm{p}=0.016$ (two tailed Fisher exact probability test); older groups: 28/31 control infants $v 13 / 24$ VLBW infants, $\mathrm{p}=0.004$ ). Figure $1 \mathrm{~B}$ shows the corresponding data, with the nine children who had any abnormalities on ultrasound excluded from the VLBW group. This reanalysis shows no significant differences between the VLBW group and the control group, although there is still a higher percentage of the control group showing the significant VEP at both ages (younger VLBW group 8/11, older group $10 / 15, \mathrm{p}=0.329$ and 0.09 respectively).

Of the four infants with a very abnormal ultrasound scan, none who completed testing showed a significant OR-VEP on any visit in the first three months. Two of these children were tested on six occasions within the first 4.5 months after term and, although they completed testing, did not show any 
significant OR-VEP on any occasion. All four of these children showed neurological abnormalities at 2 and 3 years of age, including cortical blindness in one instance.

Of the other five infants with minor ultrasound abnormalities initially, two showed significant OR-VEP responses at both 4 and 8 reversals/second, two showed significant 4 reversal/ second responses and non-significant 8 reversal/second responses initially (but a significant $8 \mathrm{reversal} / \mathrm{second}$ OR-VEP at 7 months of post-term age), and one showed nonsignificant responses initially at 4 reversals/second but significant responses at both reversal rates by 4 months of age. The two infants showing the significant 8 reversal/second response at 7 months could be considered to have a delayed cortical onset compared with normal development. This result suggests sufficient plasticity in brain development in this early period that, in cases where the initial minor abnormalities on ultrasound examination were associated with delay in cortical development indicated by the VEP, this delay was overcome within the first year. None of these five children showed significant neurological problems at 2 or 3 years of age.

\section{DISCUSSION}

The healthy premature group without ultrasound abnormalities and the control group did not show any consistent significant differences in the time of onset of orientation selective VEP responses when tested at the same age after term. These very early indicators of cortical functioning suggest that cortical development had not been accelerated by the additional visual experience of up to 16 weeks before term for the healthy premature infants. This contrasts somewhat with results we obtained for the same infants at 4-6 weeks of post-term age by measuring attentional shifts of the head and eyes. ${ }^{35}$ In this paradigm, the infant shifted fixation from a foveally fixated target in the centre of the visual field to a target that appeared in the peripheral field to either the left or right. We found significantly faster saccadic shifts in the healthy premature group than in the term infants matched for postconceptual age. However, this significant difference between the groups had disappeared by 3 months of post-term age. We suggest that this evidence of early acceleration in head and eye control for shifts of fixation, seen at around 1 month of post-term age in the premature group, may be due to superior motor control of the head and neck and a more stable alert state in the premature infants as the result of their extra visual and visuomotor experience, compared with infants in the term group.

For infants with abnormalities on ultrasound, there is delay or failure to show normal development of the cortex or related structures, as gauged by these VEP marker tests. In general, the severity of the abnormality on ultrasound correlated with the results from the early VEP testing. However, it remains to be investigated whether infants with delayed onset of cortical functioning, as indicated by their early OR-VEP measures, but normal general neurological development show more subtle deficits at a later stage of visual, cognitive, and motor development. We are currently investigating in more detail the correlation between MRI findings and early indicators of cortical functioning in a group of very premature infants with potential perinatal damage, including detailed neurological, visual, and visuocognitive follow up to 5 years of age.

In conclusion, the OR-VEP is a test that can be carried out in the newborn period for both term and preterm infants and is a prognostic indicator of onset of normal or abnormal cortical functioning. Although used here for visual development, it can probably be used as a measure of general brain development and recovery from premature birth and the sequelae of a very low birth weight. Further studies are necessary to pinpoint the particular cortical and subcortical circuits (including specific areas within basal ganglia) that are critical for normal development of the OR-VEP in normal development and under what circumstances of perinatal brain damage alternative areas and circuits become active, given the extensive plasticity of the developing visual brain.

\section{ACKNOWLEDGEMENTS}

This work was supported by programme grants to the Visual Development Unit from the Medical Research Council, UK. We thank all those in the Visual Development Unit and the Rosie Maternity Hospital (both medical staff and volunteer families) for their cooperation.

\section{Authors' affiliations}

J Atkinson, S Anker, S Rae, F Weeks, O Braddick, Visual Development Unit, Psychology Department, University College London, UK

J Rennie, Department of Paediatrics, King's College, London, UK

\section{REFERENCES}

1 Bronson GW. The postnatal growth of visual capacity. Child Dev 1974;45: 873-90.

2 Atkinson J. Human visual development over the first six months of life. A review and a hypothesis. Hum Neurobiol 1984;3:61-74.

3 Braddick OJ, Atkinson J, Wattam-Bell J. Development of visual cortical selectivity: binocularity, orientation, and direction of motion. In: von Euler C, ed. Neurobiology of early infant behaviour: Wenner-Gren symposium series. London: Macmillan, 1989:165-72.

4 Atkinson J, Van Hof-van-Duin J. Assessment of normal and abnormal vision during the first years of life. In: Fielder A, Bax M, eds. Management of visual handicap in childhood. London: MacKeith Press, 1993.

5 Braddick OJ. Orientation- and motion-selective mechanisms in infants. In: Simons K, ed. Early visual development: normal and abnormal. New York: Oxford University Press, 1993:163-77.

6 Braddick OJ, Atkinson J, Hood B. Striate cortex, extrastriate cortex, and colliculus: some new approaches. In: Vital-Durand F, Braddick OJ, Atkinson J, eds. Infant vision. Oxford: Oxford University Press, 1996:203-20.

7 Nalin A, Baraldi P, Ferrari F, et al. Visual function in term newborn-infants and preterm neonates (at the same conceptional age). Journal of Pediatrics 1981;98:858.

8 Norcia AM, Tyler CW, Piecuch RE, et al. A new look at visual acuity in premature infants [abstract]. Pediatr Res 1985;19:A368.

9 Placzek M, Mushin J, Dubowitz LMS. Maturation of the visual evoked-response and its correlation with visual-acuity in preterm infants. Dev Med Child Neurol 1985;27:448-54.

10 Ellingson RJ. Development of visual evoked-potentials and photic driving responses in normal full term, low-risk premature, and trisomy-21 infants during the 1 st year of life. Electroencephalogr Clin Neurophysiol 1986;63:309-16.

11 Pryds O, Trojaborg W, Carlsen J, et al. Determinants of visual evoked-potentials in preterm infants. Early Hum Dev 1989;19:117-25.

12 Mast J, Victor JD. Rapid (sweep) visual evoked-potential assessment of visual development in normal, premature, and developmentally delayed infants. Ann Neurol 1989;26:430.

13 Grose J, Harding G. The development of refractive error and pattern visually evoked-potentials in preterm infants. Clinical Vision Sciences 1990:5:375-82.

14 Brown B, Varma M, Vohr BR, et al. Maturation of vision evoked-response (VER) wave forms in premature (PT) and full-term (FT) infants tested at term [abstract]. Pediatr Res 1991;29:A357.

15 Harding GFA, Rudduck G. Electrophysiological correlates of visual development in premature and full-term infants. Int J Psychophysiol 1994;18:111.

16 Roy MS, Barsoumhomsy M, Orquin J, et al. Maturation of binocular pattern visual-evoked potentials in normal full-term and preterm infants from 1 to 6 months of age. Pediatr Res 1995:37:140-4.

17 Shepherd AJ, Saunders KJ, McCulloch DL, et al. Maturation of the flash visual evoked potential in premature infants. Invest Ophthalmol Vis Sci 1996;37:3342.

18 Tsuneishi S, Casaer P, Fock JM, et al. Establishment of normal values for flash visual-evoked potentials (VEPs) in preterm infants: a longitudinal study with special reference to 2 components of the $\mathrm{N} 1$ wave. Electroencephalogr Clin Neurophysiol 1995;96:291-9.

19 Sawaguchi K, Ogawa T. Component wave analysis of flash visual evoked potentials in preterm infants. Electroencephalogr Clin Neurophysiol 1998;108:62-72.

20 Ekert PG, Keenan NK, Whyte HE, et al. Visual evoked potentials for prediction of neurodevelopmental outcome in preterm infants. Biol Neonate 1997;71:148-55.

21 Shepherd AJ, Saunders KJ, McCulloch DL, et al. Prognostic value of flash visual evoked potentials in preterm infants. Dev Med Child Neurol 1999;41:9-15

22 Atkinson J, Braddick OJ. Acuity, contrast sensitivity and accommodation in infancy. In: Aslin RN, Alberts JR, Petersen MR, eds. The development of perception. New York Academic Press, $1981 ; 2$ :245-78.

23 Pike A, Marlow N, Reber C. Maturation of the flash visual evoked potential in preterm infants Early Hum Dev 1999;54:215-22. 
24 Pryds O, Greisen G, Trojaborg W. Visual evoked-potentials in preterm infants during the 1st hours of life. Electroencephalogr Clin Neurophysiol 1988;71:257-65.

25 Braddick OJ, Atkinson J, Wattam-Bell J, et al. Characteristics of orientation-selective mechanisms in early infancy. Invest Ophthalmol Vis Sci 1989;30(suppl):31.

26 Braddick OJ, Wattam-Bell J, Atkinson J. Orientation-specific cortical responses develop in early infancy. Nature 1986;320:617-19.

27 Mercuri E, Atkinson J, Braddick O, et al. Visual function and perinatal focal cerebral infarction. Arch Dis Child Fetal Neonatal Ed 1996;75:F76-81.

28 Mercuri E, Atkinson J, Braddick $O$, et al. Visual function in full term infants with hypoxic-ischaemic encephalopathy. Neuropediatrics 1997;28:155-61.

29 Mercuri E, Braddick O, Atkinson J, et al. Orientation-reversal and phase-reversal visual evoked potentials in full-term infants with brain lesions: a longitudinal study. Neuropediatrics 1998;29:1-6.
30 Mercuri E, Atkinson J, Braddick $O$, et al. Basal ganglia damage in the newborn infant as a predictor of impaired visual function. Arch Dis Child Fetal Neonatal Ed 1997;77:F111-14.

31 Mercuri E, Haataja L, Guzzetta A, et al. Visual function in term infants with hypoxic-ischaemic insults: correlation with neurodevelopment at 2 years of age. Arch Dis Child Fetal Neonatal Ed 1999;80:F99-104.

32 Van-Hof-Van-Duin J, Mohn G. The development of visual acuity in normal full-term and preterm infants. Vision Res 1986;26:909-16.

33 Weinacht S, Kind C, Monting JS, et al. Visual development in preterm and full-term infants: a prospective masked study. Invest Ophthalmol Vis Sci $1999 \cdot 40: 346-53$

34 Wattam-Bell J. Analysis of infant visual evoked potentials (VEPs) by a phase-sensitive statistic [abstract]. Perception 1985;14:A33

35 Atkinson J, Braddick OJ, Anker S, et al. Visual development in the VLBW infant. Transactions of the IVth European Conference on Developmental Psychology, University of Stirling. Stirling: European Society for Developmental Psychology, 1990: 193.

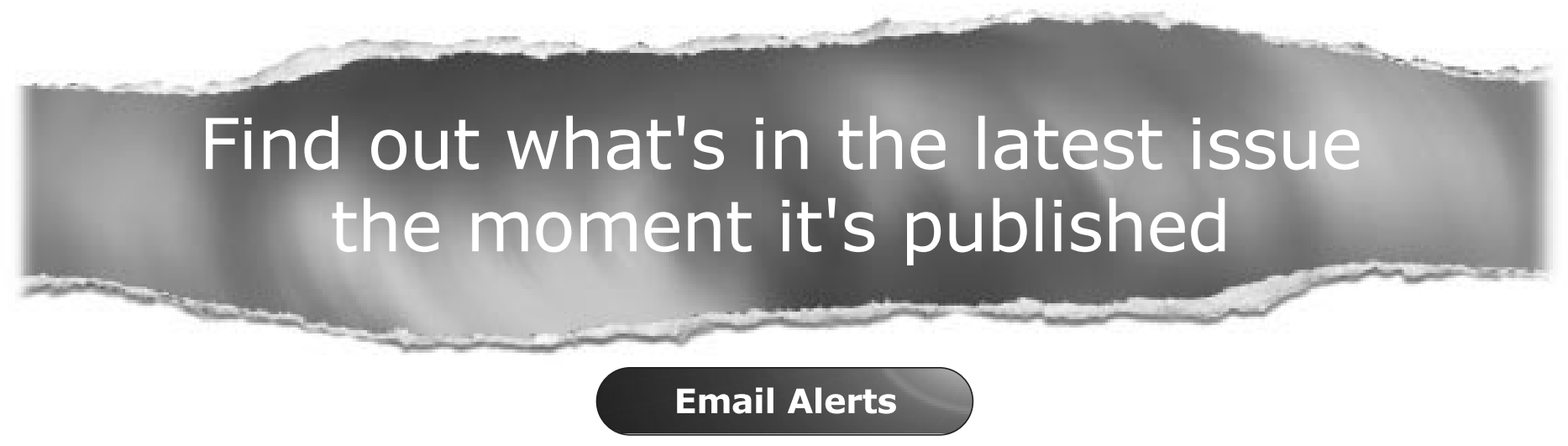

Sign up to receive the table of contents by email every month. You can select from three alerts: Table of Contents (full), TOC Awareness (notice only); Archives of Disease in Childhood related announcements.

www.archdischild.com 